\title{
Promoviendo la Formulación de Buenas Preguntas en la Clase de Biología en Secundaria: una propuesta didáctica a partir de situaciones problema
}

\section{Promoting the Formulation of Good Questions in Secondary Education Biology Classes: a pedagogical proposal based on problem situations}

\author{
Alejandra Rojas Conejera ${ }^{1}$ \\ Carol Joglar ${ }^{2}$ \\ Roxana Jara ${ }^{3}$ \\ 'Universidad de O'Higgins, Escuela de Educación, Rancagua, Chile. \\ Autora correspondente: romirojasc@ug.uchile.cl \\ ${ }^{2}$ Universidad de Santiago de Chile, Santiago de Chile, Chile. \\ ${ }^{3}$ Pontificia Universidad Católica de Valparaíso, Valparaíso, Chile.
}

Resumen: Es importante discutir sobre estrategias para desarrollar la competencia en formulación de buenas preguntas en la clase de ciencias en los estudiantes, puesto que éstas adquieren un rol protagónico en los procesos de enseñanza y aprendizaje. Por esto, instalar estrategias concretas que promuevan la formulación de buenas preguntas, se plantea como un desafío para la investigación en didáctica de las ciencias. En este artículo, se analiza cómo las actividades que utilizan situaciones problema, favorecen la formulación de buenas preguntas en el discurso escrito del estudiantado de la escuela secundaria. Estas actividades se realizaron a lo largo de cuatro sesiones de clases, durante el desarrollo de la unidad didáctica de estructura y función de la membrana plasmática. Los resultados muestran que el estudiantado participante de esta investigación presenta mejoras en las preguntas abiertas que formula, aumentando progresivamente el nivel cognitivo de sus preguntas, según transcurre la unidad didáctica.

Palavras clave: Enseñanza de la biología; Enseñanza secundaria;Técnicas de cuestionamiento; Proceso de enseñanza-aprendizaje.

\begin{abstract}
As students continue to take on more autonomy in teaching and learning processes, it is important to discuss strategies for developing their skills in formulating good questions. Indeed, instilling concrete strategies for students to craft good questions is a current challenge in science education research. In this article, we analyze how written discourse activities based on problem situations favor good question formulation among secondary school students. These activities were carried out over four class sessions during the development of a basic science education Program Unit, "Structure and Function of the Plasma Membrane". Results show that student participation under these research conditions progressively improved the cognitive level of questions formulated as the Program Unit progressed.
\end{abstract}

Keywords: Biology teaching; High school; Scientific literacy; Questioning techniques; Teaching-learning process.

Recibido en: 18/05/2019

Aprobado en: 01/04/2020 


\section{Introducción}

Actualmente es relevante que el profesorado conozca estrategias didácticas que planteen escenarios propicios para la formulación de buenas preguntas en los estudiantes de secundaria, pues, es reconocido el rol que éstas cumplen en la promoción de mejores aprendizajes científicos escolares y el desarrollo del pensamiento científico de los aprendices. Por esto, que el estudiante pueda formular buenas preguntas en las clases de ciencias es fundamental, ya que se relaciona con el planteamiento de problemas, la curiosidad por aprender ciencias y la capacidad de cuestionamiento.

\section{Antecedentes que Sustentan la Investigación}

A partir de discusiones teóricas desde el campo disciplinar de la Didáctica de las Ciencias, así como desde organizaciones internacionales como Organisation for Economic Co-operation and Development (2006) y UNESCO (2009), se reconoce que la educación científica del siglo XXI debe promover la alfabetización científica y tecnológica, para que el estudiantado aprenda ciencias, favoreciendo el desarrollo de opiniones sustentadas del mundo natural, científico y tecnológico (CAMACHO GONZALEZ; QUINTANILLA GATICA, 2008; JARA CAMPOS, 2012; JOGLAR, 2014; ZOHAR, 2008). Por esto último, se deben identificar las relaciones entre la visión epistemológica, la enseñanza y el aprendizaje de las ciencias que tiene el profesorado, pues si esta visión es menos tradicional, podrían promover con menor dificultad el desarrollo de competencias científicas $y$, consecuentemente, la alfabetización científica de sus estudiantes (MARTíNEZ GALAZ; GONZÁLEZ WEIL, 2014).

En el contexto educacional chileno, donde se realiza esta investigación, los antecedentes empíricos indican que se deben aumentar los esfuerzos para promover el desarrollo de competencias científicas en el estudiantado. Para ilustrar esto, la evaluación del Programme for International Student Assessment (PISA) realizada en el año de 2015 muestra en sus resultados que un tercio del estudiantado chileno no tiene las competencias científicas mínimas para desenvolverse en una sociedad globalizada, existiendo brechas socioeconómicas y de género significativas, cuestión relevante al momento de analizar los resultados (CHILE, 2016).

En este sentido, las políticas educativas en Chile, suscitaron una reforma curricular que se implementa en escuelas primarias y secundarias desde el año 2015, siendo uno de los ejes centrales es el desarrollo de habilidades de investigación científica, como la observación y la formulación de preguntas, considerando lo anterior como un elemento clave para el desarrollo de competencias que permitan al estudiantado comprender el mundo natural (CHILE, 2013). Esta perspectiva, se condice con lo que plantean Izquierdo et al. (1999) y Roca Tort (2005), cuando indican que la formulación de buenas preguntas jugaría un rol fundamental en la educación científica, porque permitiría a los estudiantes hablar, pensar y cuestionar la información con la que conviven a diario. Así, las buenas preguntas se convierten en una competencia fundamental para poder problematizar el conocimiento científico escolar, que el profesorado debe promover en sus estudiantes a lo largo de toda 
su trayectoria estudiantil, pudiendo superar modelos tradicionales de la enseñanza de la ciencia que actualmente prevalecen en las aulas y que se centran en la transmisión de contenidos con énfasis en lo memorístico (JARA CAMPOS, 2012).

Desde la evaluación docente en Chile, se puede identificar que la mayoría de las interacciones que realizan los profesores de ciencias, no promueven el desarrollo de pensamiento, y se sitúan desde lo memorístico-procedimental, lo que indica que las preguntas que realiza el profesorado están enfocadas en conseguir respuestas correctas y no en la problematización del conocimiento. En este sentido, sólo un $20 \%$ del profesorado de Biología que participó de la evaluación docente en Chile en el año 2010, alcanzó buenos niveles en ámbito de las interacciones pedagógicas (MANZI; GONZÁLEZ; SUN, 2011). Esto coincide con lo discutido por Blosser (2000), quien indica que el $60 \%$ de las preguntas que realiza el profesorado de ciencias están centradas en la memorización de conceptos y sólo el $20 \%$ a la estimulación del pensamiento. En consecuencia, la evidencia disponible revela que existen dificultades para formular preguntas en el profesorado y también para los estudiantes de secundaria del país, sobre todo en aspectos relacionados al desarrollo de competencias y alfabetización en ciencias.

Con relación a las nociones que describen qué es una buena pregunta, los autores plantean que que una buena pregunta es aquella que posea grados de apertura, que permita vincular las teorías y las explicaciones científicas con las ideas que el estudiante posee (CHIN; BROWN, 2002; MÁRQUEZ BARGALLÓ; ROCA TORT, 2006; ROCA TORT, 2005), que estimule la creatividad (JOGLAR, 2014) y la metacognición (CHIN; CHÍA, 2004).

Asimismo, que el estudiantado pueda formular buenas preguntas implica que el profesorado haya desarrollado esta competencia, y que éste pueda explicitarla en su enseñanza. Por último, el objetivo general de la investigación fue comprender cómo se promueven mejoras en el proceso de formulación de buenas preguntas en el estudiantado en clases de biología, durante la implementación de una unidad didáctica sobre la enseñanza de estructura y función de la membrana plasmática, que utiliza actividades con situaciones problema. En tanto los objetivos específicos planteados (1) Describir cómo la naturaleza de la actividad que utiliza situaciones problema, orienta el pensamiento hacia la elaboración de buenas preguntas en el estudiantado y (2) Caracterizar las preguntas elaboradas por el estudiantado según la naturaleza de la actividad, durante el transcurso de la unidad didáctica sobre la enseñanza de estructura y función de la membrana plasmática.

\section{Marco Teórico}

Las buenas preguntas orientan las relaciones entre modelos de estudio en la ciencia escolar, mediante hechos o fenómenos relacionados al conocimiento científico (CHIN; OSBORNE, 2008; ROCA TORT; MÁRQUEZ BARGALLÓ; SANMARTÍ PUIG, 2013). En tanto, los procesos dialógicos ligados a la pregunta en el aula, pueden ser un espacio fructífero para el devenir de los cuestionamientos y las dudas, propias de la naturaleza del conocimiento científico y la búsqueda de nuevos aportes para el mismo (CAMARGO; LINDEMEYER; RAMOS, 2011), fomentando así, la construcción de preguntas de alto nivel cognitivo, la metacognición y el trabajo investigativo en los estudiantes (CHIN; CHÍA, 2004). Por lo anterior, se considera 
esencial la reflexión sobre el valor didáctico de las buenas preguntas y cómo las interacciones comunicativas que ocurren en las clases pueden aportar o no, al proceso de enseñanzaaprendizaje.

\section{Las preguntas del estudiantado en la clase de ciencias}

La investigación en Didáctica de las Ciencias, indica que el estudiantado realiza pocas preguntas en el aula de ciencias y que, cuando las hace, éstas son generalmente cerradas, siendo muy escasas las relacionadas a la comprensión del conocimiento científico (BLOSSER, 2000; CHIN, 2007; GRAESSER; McMAHEN; JOHNSON, 1994). Según Malvaez, Joglar y Quintanilla (2013) las ideas de los estudiantes para el uso de las preguntas están centradas en la recopilación de información desde un enfoque totalmente procedimental. En lo que se refiere al diseño y formulación de las preguntas de los estudiantes, por lo general son de carácter deficiente en su formulación, no conectadas con las nociones científicas trabajadas y relativas a situaciones de gestión de la escuela.

Ahora bien, no es tan sencillo comprender cuándo una pregunta destaca como una buena pregunta, sin embargo, existen algunas características y tendencias que se pueden mirar para ir definiendo este punto. Por ejemplo, las preguntas abiertas destacan como posibles mediadoras que estimulen el desarrollo de pensamiento de orden superior (ZOHAR, 2008), además de relacionarse con distintos aspectos de la explicación científica (MÁRQUEZ BARGALLÓ; ROCA TORT, 2006; ROCA TORT, 2005). Esto, según Roca Tort, Márquez Bargalló y Sanmartí Puig (2013) llevaría al estudiantado a formular preguntas desde la descripción, explicación causal, la generalización, las pruebas para la comprobación, la predicción, la gestión y la evaluación. Además, una buena pregunta, tendría que propiciar el vínculo entre la teoría científica con las teorías implícitas que el estudiante posee (CHIN; BROWN, 2002), estimulando así su creatividad, curiosidad y la modelización de los fenómenos científicos que aprende (JOGLAR, 2014). Por último, una buena pregunta tendría que llevar al estudiante a procesos metacognitivos y facilitar una conexión con el trabajo investigativo (CHIN; CHÍA, 2004). Más aún, la realización de buenas preguntas permitiría a los estudiantes conectar conceptos nuevos, con sus intereses, permitiendo a compañeros y docentes a que conozcan sus pensamientos y argumentos (AGUIAR; MORTIMER; SCOTT, 2010; SILVESTRI, 2006).

Por lo anterior, no podemos discutir el valor didáctico que tiene la formulación de buenas preguntas en contexto de enseñanza y aprendizaje de las ciencias, pues también está relacionado con la capacidad de proveer de explicaciones en función del conocimiento que van trabajando y de la asociación con el conocimiento científico (MÁRQUEZ BARGALLÓ; ROCA TORT, 2006). Sin embargo, es necesario que el estudiantado distinga las buenas de las malas preguntas, y eso es posible sólo si se propician espacios didácticos para fomentar la formulación de buenas preguntas. Las buenas preguntas serán el resultado de discrepancias o disonancias cognitivas que llevarán a expandir el conocimiento científico en el estudiantado (CHIN; BROWN, 2002), por lo tanto, es primordial que el profesorado vaya generando espacios que estimulen la formulación de buenas preguntas en sus estudiantes, a partir de estrategias didácticas consistentes. 
En este sentido, Chin (2007) muestra que una estrategia que podría promover la competencia de elaborar buenas preguntas en el estudiantado, es precisamente que el profesorado haga buenas preguntas en sus clases, sin embargo, es sustancial proponer espacios intencionados que sirvan de herramienta para que el estudiantado realice buenas preguntas. Por esto, es relevante que el profesorado formule preguntas que acerquen a los estudiantes a una comprensión de la naturaleza de la ciencia situada en buscar respuestas a las problemáticas que nos afectan en la actualidad (ROCA TORT, 2005), pues la capacidad que tengan los profesores de plantear buenas preguntas en las clases de biología impactará en los procesos cognitivos de los estudiantes con los que trabaja (CHIN; BROWN, 2002). Para clasificar los tipos de preguntas, se han levantado distintas propuestas de análisis, Amos (2002) agrupa las preguntas en abiertas y cerradas; Roca Tort, Márquez Bargalló y Sanmartí Puig (2013) definen como preguntas cerradas cuando sólo una respuesta es posible y correcta, y preguntas abiertas, si existen varias posibilidades de respuesta. Asimismo, Lawson (2002) presenta una propuesta basada en el trabajo de indagación, en donde releva los modelos teóricos de lo que observan y aprenden los estudiantes, en donde uso de las preguntas presenta como estrategia para trabajar dichas teorías (MÁRQUEZ BARGALLÓ; ROCA TORT, 2006).

\section{Las situaciones problema como estrategia didáctica para la elaboración de buenas preguntas}

Un problema se define como un cuestionamiento, vacilación o dilema, al que se debe buscar una solución (BARELL, 1999); así, para este autor, las situaciones problema deben tener ciertas características como: ser situaciones abiertas a posibles preguntas, no ser estructuradas, despertar la curiosidad y ser significativas para los estudiantes. En ese sentido, Pozo y Angón (1998) refuerzan esta idea e indican que una situación problema debe abrir espacios para búsqueda de estrategias pertinentes para su solución, no sólo desde el punto de vista disciplinar, sino que también desde el mundo cotidiano los estudiantes; por esto, los autores plantean que un problema científico escolar tiene por objetivo generar conceptos, procedimientos y actitudes propios de la ciencias, pero además, debe responder a situaciones propias y cotidianas del mundo natural y la tecnología. De acuerdo a lo anterior, se han investigado propuestas para promover mejoras en la formulación de preguntas en los estudiantes universitarios (JESUS; MOREIRA, 2009), mostrando como la estrategia didáctica de la utilización de problemas, que históricamente se ha utilizado para generar respuestas y explicaciones por parte de los estudiantes, puede promover mejorar en los tipos de preguntas que se formulan.

En esta investigación, se utiliza la estrategia didáctica de situaciones problema y se diseñan actividades contextualizadas desde las cuestiones socio-científicas, noticias científicas, historia de la ciencia y actividades prácticas. Lo anterior se detalla en el Cuadro 1 , en el que se diferencian teóricamente las temáticas que utilizan situaciones problemas en las actividades propuestas para esta investigación. 
Cuadro 1 - Temáticas de contextualización de actividades que utilizan situaciones problema

\begin{tabular}{|c|c|}
\hline $\begin{array}{l}\text { Cuestiones } \\
\text { Sociocientíficas }\end{array}$ & $\begin{array}{l}\text { Las cuestiones sociocientíficas corresponden a temáticas relacionadas con la Ciencia y Tecnología, que entienden la } \\
\text { ciencia como una actividad humana con controversias y dilemas en su construcción, que incluyen análisis de situ- } \\
\text { aciones relativas a lo ético y lo moral de implicancia en lo público, encontrando en ellas alcances para la formación } \\
\text { ciudadana del estudiantado a partir de situaciones de alto impacto en la sociedad (DÍAZ-MORENO; JIMÉNEZ-LISO, } \\
\text { 2012; MARTÍNEZ-PÉREZ; PARGA LOZANO, 2013). Estas situaciones, suelen ser continuamente publicadas en medios } \\
\text { de comunicación (periódicos, internet, tv, radio, etc.), lo que resulta en una mayor visibilidad para la población } \\
\text { (MARTíNEZ-PÉREZ; PARGA LOZANO, 2013), y que podría ser utilizado como temáticas de interés y comunión para } \\
\text { los estudiantes en las clases de ciencia. } \\
\text { Además, las cuestiones sociocientíficas movilizan ciertas discrepancias o consensos entre distintos actores que } \\
\text { puedan estar implicados en estas problemáticas (DÍAZ-MORENO; JIMÉNEZ-LISO, 2012) y representan puntos de } \\
\text { partidas para promover reflexiones en torno a consecuencias en temáticas, socioculturales, económicos, valóricos } \\
\text { que ponen en juego visiones sobre los objetivos de la enseñanza de las ciencias (SOLBES; TORRES MERCHÁN, 2012). } \\
\text { Por otra parte, se utilizan actividades que provienen de noticias que vinculan problemáticas desde la ciencia (BLOS- } \\
\text { SER, 2000) pero no corresponden directamente a cuestiones socio-científicas. }\end{array}$ \\
\hline iencia & $\begin{array}{l}\text { El uso de la historia de la ciencia, se entiende como una estrategia didáctica que promueve clases más desafiantes, } \\
\text { que instan al diálogo y al desarrollo del pensamiento crítico en el estudiantado, que apoyarían una comprensión } \\
\text { más amplia del conocimiento científico a partir de su vinculación con temáticas culturales, éticas y políticas (MAT- } \\
\text { THEWS, 1994). Lo anterior, posibilita mostrar al estudiantado una actividad científica más humanizada, que tiene } \\
\text { aproximaciones subjetivas desde su construcción con lo cultural e histórico (SOLBES; TRAVER, 2001), enmarcado en } \\
\text { problemáticas relativas a un contexto en particular y a la resolución de problemas mediados por dichos factores. }\end{array}$ \\
\hline Actividades & $\begin{array}{l}\text { Las actividades experimentales por algunos autores denominados actividades prácticas (CAAMAÑO ROS, 2004), } \\
\text { son aquellas que conllevan ejercicios para el aprendizaje de lo metodológico, técnico e investigativo que se usan } \\
\text { para comprender procesos de la ciencia y construir conocimiento científico. También, pueden ser utilizadas para } \\
\text { generar vinculaciones con aspectos acerca de la naturaleza y filosofía de la ciencia (SANMARTí; MÁRQUEZ; GARCÍA, } \\
\text { 2002), que instaría al estudiantado a un aprendizaje conceptual y desarrollo de habilidades de pensamiento (TEN- } \\
\text { REIRO-VIEIRA; MARQUES VIEIRA, 2006), que convierten a esta estrategia didáctica como una propuesta concreta de } \\
\text { experiencias de aprendizaje significativas. }\end{array}$ \\
\hline
\end{tabular}

Fuente: elaboración de las autoras.

Según lo planteado anteriormente, se puede potenciar el uso de situaciones problema en el aula utilizando distintos contextos, como promotoras de buenas preguntas y otras competencias fundamentales para el estudiantado, como lo son las explicaciones científicas, que se van fundando de acuerdo con la búsqueda de respuestas (ROCA TORT; MÁRQUEZ BARDALLÓ; SANMARTÍ PUIG, 2013). Entonces, según lo plantean estas autoras, es importante promover en los estudiantes preguntas orientadas a la explicación causal, la generalización, las pruebas para la comprobación, la predicción, la gestión y la evaluación; esta clasificación, conlleva una opción concreta de análisis y objetivación de estas. Por consiguiente, dicha propuesta se podría promover un tránsito hacia niveles más complejos de pensamiento (ZOHAR, 2008), aportando así, de manera mucho más compleja e integral al aprendizaje de los estudiantes.

\section{Metodología}

Esta investigación fue llevada a cabo en la ciudad de Santiago de Chile, en una institución escolar pública que imparte educación primaria y secundaria, a la que asisten niños y niñas de seis a dieciocho años. Los casos en estudio fueron dos cursos de noveno grado con cuarenta y seis estudiantes cada curso, que para efectos de esta investigación serán denominados Curso $A$ y Curso $B$, cuyos estudiantes tenían un promedio de edad de quince años. La selección de la muestra se hizo bajo un criterio no probabilístico (HERNÁNDEZ SAMPIERI; FERNÁNDEZ COLLADO; BAPTISTA LUCIO, 2010). Además, el diseño utilizado en esta investigación fue el Estudio de Caso, que supone una comprensión detallada y sistemática 
del objeto de estudio como lo indica Rodríguez Gómez et al. (1996 apud SANDíN, 2003, p. 174). Los casos fueron seleccionados por conveniencia, y en la fase de generación de datos fueron considerados aspectos contextuales de los sujetos involucrados en la investigación con la finalidad de indagar en profundidad en el fenómeno (SANDíN, 2003).

\section{Instrumentos para la recolección de datos}

Se utilizaron siete actividades escritas, basadas en situaciones problema que el estudiantado tuvo que realizar en sesiones de clases consecutivas, durante el desarrollo de una Unidad Didáctica sobre Estructura y Función de Membrana Plasmática. En el Cuadro 2 se resumen aspectos sobre el objetivo, la naturaleza y la instrucción de cada una de las actividades que fueron resueltas por el estudiantado.

Cuadro 2 - Resumen de instrumentos aplicados a estudiantes para promover mejoras en la elaboración de preguntas

\begin{tabular}{|c|c|c|c|c|}
\hline $\mathbf{N}^{\circ}$ Actividad & $\begin{array}{l}\text { Nombre de la } \\
\text { Actividad }\end{array}$ & Tipo Actividad & Instrucción Actividad & Objetivo de la actividad \\
\hline 0 & $\begin{array}{l}\text { Guía de trabajo indi- } \\
\text { vidual diagnóstica. } \\
\text { Biomoléculas y salud }\end{array}$ & $\begin{array}{l}\text { Cuestión Sociocientífica: Una } \\
\text { niña de } 2 \text { años que llevaba una } \\
\text { dieta vegana, ingresada en ur- } \\
\text { gencias en hospital de Génova, } \\
\text { Italia }\end{array}$ & $\begin{array}{l}\text { Si tú fueras el Médico Tratante } \\
\text { de esta niña: ¿Qué preguntas } \\
\text { habrías hecho a los padres de } \\
\text { Ana al momento de ingresarla } \\
\text { a la urgencia para obtener infor- } \\
\text { mación que permita contribuir a } \\
\text { su diagnóstico médico? }\end{array}$ & $\begin{array}{l}\text { Formular preguntas para obte- } \\
\text { ner información sobre el diag- } \\
\text { nóstico }\end{array}$ \\
\hline 1 & $\begin{array}{l}\text { Guía de trabajo indi- } \\
\text { vidual de exploraci- } \\
\text { ón ideas previas }\end{array}$ & $\begin{array}{l}\text { Actividad práctica demostrativa: } \\
\text { osmosis en huevos }\end{array}$ & $\begin{array}{l}\text { Luego de observar deteni- } \\
\text { damente los huevos en sus } \\
\text { respectivos recipientes. ¿Qué } \\
\text { preguntas harías sobre lo que } \\
\text { observas? Formula } 3 \text { preguntas. }\end{array}$ & $\begin{array}{l}\text { Formular preguntas para obte- } \\
\text { ner información sobre el fenó- } \\
\text { meno observable. }\end{array}$ \\
\hline 2 & $\begin{array}{l}\text { Guía individual al } \\
\text { finalizar la clase }\end{array}$ & $\begin{array}{l}\text { Historia de la ciencia: Conocien- } \\
\text { do algo de historia de la Biología } \\
\text { y las primeras observaciones } \\
\text { sobre la membrana plasmática }\end{array}$ & $\begin{array}{l}\text { Formula 3: preguntas que crees } \\
\text { se hizo Ernest Charles Overton } \\
\text { sobre su observación acerca de } \\
\text { la absorción de sustancias por la } \\
\text { raíz. (JOGLAR et al., 2014) }\end{array}$ & $\begin{array}{l}\text { Formular preguntas sobre un } \\
\text { fenómeno científico. }\end{array}$ \\
\hline 3 & $\begin{array}{l}\text { Prueba, ítem de de- } \\
\text { sarrollo }\end{array}$ & $\begin{array}{l}\text { Cuestión sociocientífica: Muere } \\
\text { la niña chilena que le pidió la } \\
\text { eutanasia a la presidenta Bache- } \\
\text { let, luego de su lucha contra la } \\
\text { Fibrosis Quística }\end{array}$ & $\begin{array}{l}\text { A partir de la lectura del texto: } \\
\text { Formula } 3 \text { preguntas que le harí- } \\
\text { as a un investigador o una inves- } \\
\text { tigadora de esta enfermedad, } \\
\text { sobre posibles tratamientos }\end{array}$ & $\begin{array}{l}\text { Formular preguntas para ob- } \\
\text { tener información sobre trata- } \\
\text { mientos médicos }\end{array}$ \\
\hline 4 & $\begin{array}{l}\text { Prueba, ítem de de- } \\
\text { sarrollo }\end{array}$ & $\begin{array}{l}\text { Cuestión sociocientífica: Muere } \\
\text { la niña chilena que le pidió la eu- } \\
\text { tanasia a Bachelet, luego de su } \\
\text { lucha contra la Fibrosis Quística }\end{array}$ & $\begin{array}{l}\text { Formula 3: preguntas que le ha- } \\
\text { rías a la Ministra de Salud, pien- } \\
\text { sa en Valentina y otros niños y } \\
\text { niñas que sufren enfermedades } \\
\text { graves como estas }\end{array}$ & $\begin{array}{l}\text { Formular preguntas para iden- } \\
\text { tificar mecanismos de gestión } \\
\text { y opinión }\end{array}$ \\
\hline
\end{tabular}

Fuente: elaboración de las autoras.

\section{La Generación de los Datos}

Para poder abordar el objetivo planteado, metodológicamente el diseño de actividades y recolección de datos se dividió en 3 etapas que se detallan a continuación.

a. Observación de clases y aplicación de actividad con situación problema 0 o inicial: esta etapa duró dos semanas y consistió en la observación no participante de ocho horas de clases de biología para cada curso, en la última clase se aplicó a cada curso respectivamente, una actividad escrita individual que contenía una situación problema 0 o inicial; 
b. Talleres de planificación de unidad didáctica y actividades con situaciones problema: durante seis semanas consecutivas, se realizaron todos los días miércoles jornadas de planificación con las profesoras de biología y la investigadora. En estas sesiones, se decidió trabajar con la 'Unidad de estructura y función de la membrana plasmática', además de consensuar que las clases tendrían distintos momentos. En ese sentido, las profesoras solicitaron resguardar sus espacios de cátedra, pues necesitaban cumplir con los objetivos curriculares designados, por lo que más que diseñar una unidad didáctica que utiliza la estrategia de situaciones problemas, se diseñaron actividades escritas (en conjunto) enfocadas en situaciones problema, para ser trabajadas en cada clase;

c. Implementación de unidad didáctica y aplicación de actividades: durante cuatro semanas consecutivas, se desarrollaron las clases de la unidad didáctica de estructura y función de la membrana plasmática, en la que resguardaron momentos en la clase para que a través de las actividades, los estudiantes pudieran formular preguntas de acuerdo a las situaciones problema planteadas y a partir de esta premisa es que se analizan los resultados.

\section{Análisis de los Datos}

Para validar esta investigación se aplicaron criterios de rigor científico de validez y fiabilidad, para dar sustento al análisis de los datos. Para esto, se mencionan los criterios de validez según Rodríguez, Gil y García (1999): (1) Validez descriptiva: refiere a la precisión de los datos sin haber sido adulterados, en todas las etapas del análisis; (2) Validez interpretativa: los datos se van acotando según los marcos teóricos de referencia; (3) Validez teórica: las decisiones que toma la investigadora de esta tesis se enmarcan dentro del marco teórico referenciado desde la Didáctica de las Ciencias Experimentales; (4) Fiabilidad de transparencia y contextualización: se da cuenta detalladamente de la recogida de datos y diseño de esta investigación.

La técnica utilizada para el análisis de los datos, fue el análisis del contenido (PORTA; SILVA, 2003) que permite indagar sobre la constitución del discurso, para analizar detalladamente aspectos relacionados a la comunicación en códigos lingüísticos que disponen instrumentos escritos u orales como cuestionarios, agendas, encuestas, entrevistas, entre otros. Se analizaron los datos de dos estudios de caso que corresponde al Curso A (con veinticinco estudiantes) y al Curso $B$ (con veintitrés estudiantes), estos estudiantes fueron los que desarrollaron el total de las actividades. La categorización de las preguntas se efectuó por una especialista en el área y la investigadora.

Se obtuvo un total 685 preguntas formuladas, en cada una de las cuatro actividades detalladas anteriormente (Cuadro 2). Para esto, las preguntas formuladas se transcribieron y se categorizaron inicialmente en dos grandes grupos: preguntas cerradas y preguntas abiertas (AMOS, 2002; ROCA TORT; MÁRQUEZ BARGALLÓ; SANMARTÍ PUIG, 2013), existiendo también otras dos subcategorías como las preguntas mal formuladas y las preguntas que a pesar de ser cerradas en su formulación, tenían un gran potencial para transformarse en preguntas abiertas de alta complejidad cognitiva, que fueron denominadas para esta investigación como preguntas cerradas mejorables (ver Cuadro 3). 
Cuadro 3 - Resumen de frecuencia de preguntas cerradas ( $P C)$, preguntas cerradas mejorables $(P C M)$, preguntas abiertas (PA) y preguntas mal formuladas (PMF) del Curso $A(C A)$ y Curso $B(C B)$

\begin{tabular}{|c|c|c|c|c|c|c|c|c|c|c|c|}
\hline & \multicolumn{2}{|c|}{ Actividad 0} & \multicolumn{2}{|c|}{ Actividad 1} & \multicolumn{2}{|c|}{ Actividad 2} & \multicolumn{2}{|c|}{ Actividad 3} & \multicolumn{2}{|c|}{ Actividad 4} & \multirow[t]{2}{*}{ Total } \\
\hline & CA & CB & CA & CB & CA & CB & CA & CB & CA & CB & \\
\hline Preguntas Cerradas & 67 & 25 & 7 & 0 & 10 & 1 & 21 & 17 & 15 & 23 & 186 \\
\hline Preguntas Cerradas mejorables & 3 & 0 & 7 & 0 & 4 & 0 & 15 & 5 & 8 & 7 & 49 \\
\hline Preguntas Abiertas & 10 & 48 & 55 & 67 & 55 & 57 & 31 & 38 & 48 & 35 & 444 \\
\hline Preguntas Mal formuladas & 1 & 0 & 1 & 1 & 1 & 0 & 1 & 0 & 2 & 0 & 7 \\
\hline Total & 81 & 73 & 70 & 68 & 69 & 58 & 68 & 60 & 73 & 65 & 685 \\
\hline
\end{tabular}

Fuente: elaboración de las autoras.

A partir de esta clasificación inicial de las preguntas elaboradas por el estudiantado, se prosiguió con una categorización secundaria de las preguntas abiertas.

\section{Categorización de las Preguntas Abiertas Formuladas por el Estudiantado}

Para efectos de este análisis, se caracterizaron las 444 preguntas abiertas formuladas por el estudiantado de ambos cursos en las cuatro actividades planteadas (ver Cuadro 2). Para esto, se utiliza una propuesta de análisis de Roca Tort, Márquez Bargalló y Sanmartí Puig (2013), que elevan categorías de análisis, según lo que denominan Categorías de objetivo o demanda de la pregunta, caracterizándose en preguntas descriptivas, de explicación causal, generalización, comprobación, predicción, gestión y evaluación/opinión. Estas categorías de además de tener objetivos particulares, poseen una trayectoria desde niveles cognitivos más sencillos a otros más complejos.

Para finalizar el análisis, se compararon los tipos de preguntas formuladas en cada actividad en su trayectoria durante la Unidad Didáctica, con la intención de evidenciar los tránsitos en las preguntas formuladas desde el inicio, durante y al final. Además, se analizaron las posibles vinculaciones entre la naturaleza de la actividad y el tipo de preguntas que generaba.

\section{Resultados}

\section{Preguntas abiertas y cerradas}

A partir de la categorización inicial de preguntas abiertas y cerradas (ver Cuadro 3), se observa que el número de preguntas cerradas en el Curso A y en el Curso B, fue disminuyendo a medida que las actividades avanzaban, salvo en las dos últimas actividades para el Curso $B$ en que hubo un aumento en el número de preguntas cerradas. En contraparte, las preguntas abiertas, fueron aumentando a partir del transcurso de las actividades en ambos cursos, salvo en la actividad 4 para el Curso B, que hubo una disminución en el número de preguntas abiertas. Los resultados de la caracterización inicial, se muestran en los gráficos que siguen. 
Figura 1 - Gráficos clasificación inicial de preguntas Curso A y Curso B. Número de preguntas según actividad
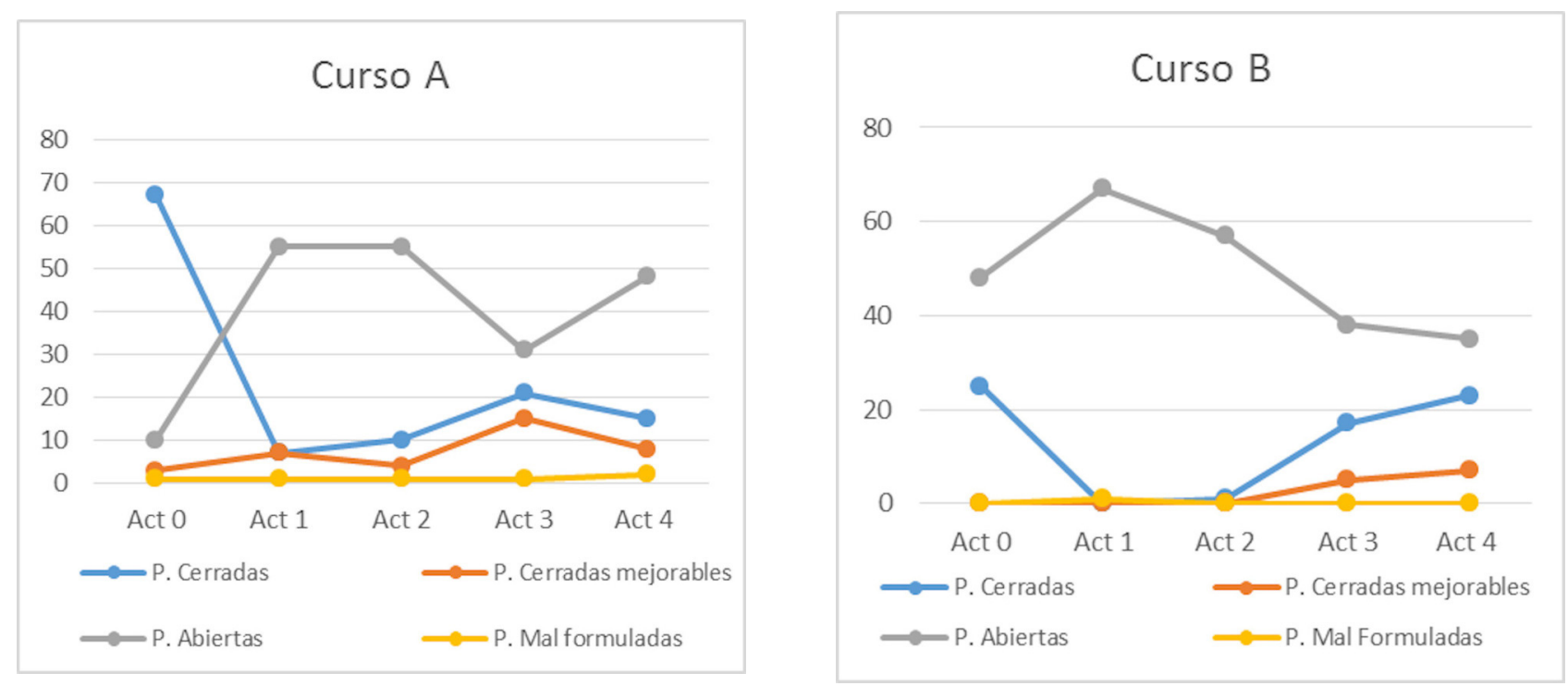

Fuente: elaboración de las autoras.

Además, en estos resultados vemos un aumento en las preguntas cerradas mejorables en el proceso, situación que podría explicarse porque los estudiantes pueden presentar mejoras en la formulación de preguntas a medida que tienen la oportunidad de practicar y pensar esta competencia con secuencias de actividades intencionadas para tal fin (FURMAN; BARRETO PÉREZ; SANMARTÍ, 2006), lo que también puede influir en su motivación por saber y preguntarse sobre el conocimiento científico (SANMARTÍ; MÁRQUEZ; GARCÍA ROVIRA, 2002). Si vemos los resultados comparativos con respecto a las preguntas abiertas, éstas fueron aumentando en número mientras desarrollaban las actividades en sesiones de clases sucesivas. Esta tendencia se hace notoria desde la actividad 1 en adelante (ver Figura 1).

\section{Preguntas abiertas}

Posteriormente, se generó una caracterización de los tipos de preguntas abiertas del Curso A y Curso B, cuyos resultados se muestran en los gráficos que siguen.

Figura 2 - Gráfico de caracterización de preguntas abiertas según número por categoría, para los instrumentos aplicados en el Curso A y Curso B
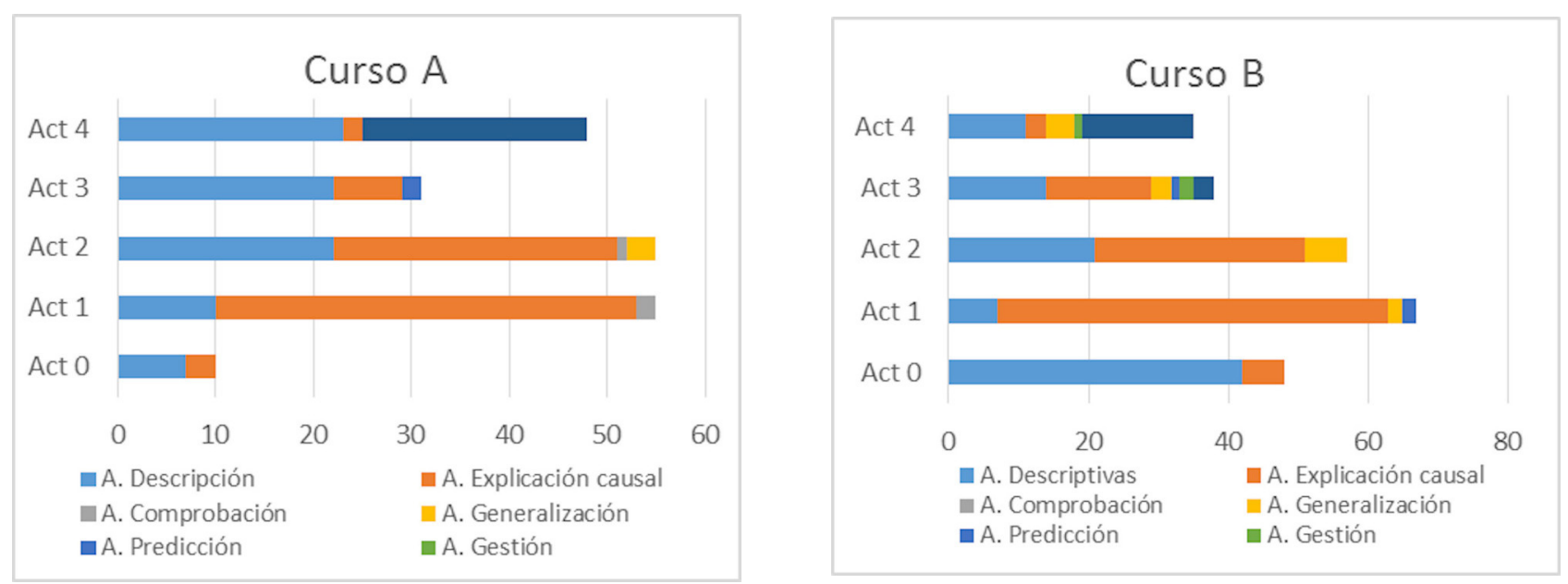

Fuente: elaboración de las autoras. 
En los resultados graficados de la Figura 2 es posible ver como el número de preguntas abiertas aumenta para ambos cursos, sucesivamente los estudiantes desarrollan las actividades en clases consecutivas, sin embargo, el número inicial de preguntas abiertas en la Actividad 0 fue mayor en el Curso B.

Con respecto al tipo de preguntas abiertas, el Curso A presenta un predominio de las preguntas descriptivas y de explicación causal en todas las actividades, lo que podría explicarse porque son las preguntas que los estudiantes más conocen y escuchan a lo largo de su trayectoria escolar. Sin embargo, con el paso de las actividades van apareciendo paulatinamente preguntas de niveles cognitivos más elevados, como las preguntas de comprobación, predicción, y evaluación, a pesar de que son siempre menos frecuentes que las descriptivas y explicación causal, excepto en la Actividad 4 en que las preguntas de evaluación igualan a las preguntas descriptivas. En el Curso B, las preguntas descriptivas y de explicación causal son las más frecuentes en las cuatro actividades, las preguntas de generalización se encuentran presentes en tres actividades y desde la Actividad 1 en adelante (excepto en la Actividad 2), pueden verse preguntas de gestión y predicción, siendo las preguntas de evaluación las terceras más frecuentes en la Actividad 4.

\section{Preguntas Abiertas Según la Naturaleza de las Actividades}

A continuación, se detalla cómo ocurre la formulación de preguntas por parte del estudiantado según la naturaleza y objetivo de la actividad. Se utilizan los códigos E, para referirnos a los estudiantes, y $C$ para referirnos a los cursos, así $E 3$ corresponde al estudiante 3, CA/CB corresponde al Curso A o Curso B respectivamente.

Según esto, podemos distinguir tres categorías:

\section{Actividades de naturaleza socio-científica y noticias científicas (0, 3 y 4):}

a. Para la actividad 0 el Curso $A$ y el Curso $B$ formularon preguntas descriptivas (E3CB: ¿Cuáles son los alimentos que consume Ana habitualmente?; E4CA: ¿Con qué clase de alimentos veganos ustedes reemplazaban los alimentos que según su dieta Ana no debe consumir?) y de explicación causal (E1CA: ¿Por qué creen que los alimentos provenientes de los animales son perjudiciales para la salud?; E12CB: ¿Por qué no le hacen consumir alimentos de animales?), existiendo diferencias entre ambos cursos con respecto a la cantidad de preguntas abiertas elaboradas. Para esta actividad el objetivo de la actividad corresponde a formular preguntas para obtener información para el diagnóstico, lo que se condice con los tipos de preguntas elaboradas y que podría explicarse, por ser la primera vez que desarrollaban este tipo de actividades y por ser este tipo de preguntas las que más comúnmente se utilizan.

b. Para la actividad 3, los resultados fueron un tanto dispares en cada curso, los estudiantes del Curso A y B formularon preguntas descriptivas (E2CA: ¿Cómo se podría lograr que pase el cloro a través de la membrana?; E7CB: ¿Cómo se puede detener esta enfermedad?), de explicación causal (E1CB: ¿Por qué suele afectar más a niños y adolescentes más que en adultos?; E2CA: ¿Por qué afecta principalmente a los pulmones?) y predictivas (E3CA: ¿Qué acción puede provocar este malfuncionamiento de la proteína integral?). En el Curso B hubo mayor variedad, pues también 
elaboraron preguntas de generalización (E2CB: ¿Cómo se podría tratar las proteínas para su óptimo funcionamiento?), gestión (E6CB: ¿Cómo se podría prevenir que se intervenga el paso del ión cloro?) y evaluación (E11CB ¿Qué se le ocurre que podría ser la cura para esta enfermedad si la tuviera?). El objetivo de esta actividad fue formular preguntas que buscan identificar terapias, lo que podría explicar el origen de preguntas de gestión y evaluación respectivamente.

c. Para la actividad 4, se muestra una tendencia hacia las preguntas de evaluación en ambos Cursos (E15CA: ¿Qué tan afectada se ve usted como ministra por esta enfermedad que atormenta a miles de niños?; E10CB: ¿Qué haría para mejorar la vida de las personas que padecen esta enfermedad?) en ambos cursos, que incluso igualan y/o superan a las descriptivas (E1CA: ¿Cuántas personas aproximadamente tienen enfermedades de este estilo?; E11CB: ¿Qué calidad de atención tienen estos niños que padecen de esta enfermedad?), existiendo también algunas preguntas de generalización (E10CB: ¿Cómo podría el medio ambiente afectar esta enfermedad?) y gestión (E16CB: ¿Cómo se puede impedir?) en el Curso B. Lo anterior podría explicarse por el objetivo de la actividad que solicitaba formular preguntas que buscan identificar mecanismos de gestión, y su contexto incluía preguntar a una autoridad del país, sobre una situación vivida por una joven de edad similar a los estudiantes, lo que podría explicar una fuerte tendencia éstos a plantear preguntas evaluativas.

2. Actividad de naturaleza práctica (1): En esta actividad, existe tendencia en ambos cursos hacia la elaboración de preguntas de explicación causal (E2CA: ¿Por qué cambia la contextura, es decir, la forma de los huevos?; E3CB: ¿Por qué cambió el tamaño del huevo con la sal y con el suero?), con una menor cantidad de preguntas descriptivas (E14CB: ¿Cuánto tiempo tiene que estar en el frasco para quedar así?; E1CA: ¿Qué cambio químico tiene que ocurrir para que quedaran así?). Además, en el Curso A se formulan algunas preguntas de comprobación (E5CA: ¿Podrían volver a su estado natural si se revierten las causas del estado actual?) y en el Curso B preguntas de generalización (E10CB: ¿Cómo varío su tamaño al estar en soluciones distintas?) y predicción (E11CB: ¿Cómo influye las sustancias en la que se sumergen los huevos?). El objetivo de esta actividad fue formular preguntas sobre el fenómeno, esto explicaría la gran cantidad de preguntas de explicación causal elaboradas por los estudiantes.

3. Actividad de historia de la ciencia (2): En esta actividad, se evidenció en ambos cursos una tendencia similar a la de la actividad 1, las preguntas elaboradas por los estudiantes en este caso fueron preguntas descriptivas (E2CB: ¿Qué tiene la raíz que permite mayor facilidad para absorber y excretar algunas sustancias?) de explicación causal (E2CA: ¿Por qué unas sustancias se absorben y otras excretan de las células vegetales?) y generalización (E13CA: ¿Cómo las plantas conseguían absorber algunas sustancias y excretar otras?) en ambos cursos, existiendo una pequeña cantidad de preguntas de comprobación en el curso A (E22CA: ¿Cómo lo puede comprobar?). EI objetivo de esta actividad era que el estudiantado elaborara preguntas sobre un fenómeno histórico, que a su vez tendría relación con el objetivo de la actividad 1 y por ende, esto podría explicar la similitud en los tipos de preguntas elaboradas por los estudiantes. 
Las preguntas formuladas por el estudiantado en las últimas actividades revelan cómo se generaron preguntas de casi todos los tipos de categorías y niveles cognitivos, excepto preguntas de comprobación que, en términos globales fueron los tipos de preguntas menos se formularon.

\section{Discusión}

\section{Resguardo de espacios para la promoción de buenas preguntas}

En esta investigación se refuerza la idea de la importancia de resguardar espacios intencionados para el diseño de actividades con un enfoque particular (como los detallados anteriormente) en las clases de ciencia. No obstante, se presenta como el objetivo, la instrucción y la naturaleza de la actividad, también otorgan información sobre la intencionalidad de las actividades y estimulación el pensamiento hacia direcciones determinadas en el estudiantado. Un ejemplo de lo anterior se puede ver en la actividad 4, cuyo objetivo buscaba generar preguntas relativas a mecanismos de gestión, con una naturaleza socio-científica, en la que las preguntas de evaluación/opinión generadas por el estudiantado ocupan un lugar relevante.

\section{Actividades con objetivos claros}

El profesorado que planifica sus distintas actividades con un objetivo claro y una instrucción específica, entregará a sus estudiantes propuestas que los lleven a direccionar y desarrollar su pensamiento hacia niveles específicos, según se va guiando el trabajo en clases. Por otro lado, es relevante mencionar que cada curso tuvo profesoras diferentes, lo que permite inferir que esta estrategia se comportaría como precursora en la formulación de buenas preguntas en los estudiantes, a pesar de las posibles diferencias en la enseñanza de las profesoras.

\section{Uso de situaciones problema como estrategia didáctica para la promoción de buenas preguntas en el estudiantado}

Otro aspecto a mencionar, es que los resultados de esta investigación entrega estrategias didácticas concretas que permitan estimular la formulación de buenas preguntas por parte del estudiantado así como el desarrollo del pensamiento en niveles cognitivos más altos (ZOHAR, 2008) empleando una estrategia relativamente conocida y utilizada por el profesorado en sus clases como lo son las situaciones problema, que habitualmente se enmarcan en la generación de respuestas y construcción de explicaciones, pero que en este caso aparece como una oportunidad para desarrollar la competencia de preguntar en el estudiantado. Otros estudios como el de Jesus y Moreira (2009), que utilizan problemas en clases de química en estudiantes universitarios, muestran un avance en la generación de preguntas abiertas que, si bien en su mayoría fueron de bajo nivel cognitivo, aumentaron en número y relación hacia el problema con el que trabajaron e indican sugerencia de mejoras en el aprendizaje desde la vinculación con las preguntas. 
Igualmente, es posible destacar ciertas tendencias con respecto a la naturaleza de las actividades y los tipos de preguntas abiertas que generan. Por ejemplo, en el Cuadro 2, se ve que en las actividades ( 0,3 y 4 ) que utilizan cuestiones socio-científicas, hay tendencia hacia la formulación de preguntas de generalización, gestión y marcadamente hacia las preguntas de evaluación-opinión, esto último podría explicarse al tratarse de problemáticas en el ámbito de lo social, lo ético y que por ende podrían ser del interés del estudiantado. La actividad de naturaleza práctica (1), generó mayormente preguntas de explicación causal y un número inferior de preguntas de comprobación, esto también podría estar relacionado a la naturaleza de la actividad práctica que promovería que el estudiantado busque como explicar y comprobar el fenómeno observado. Así, la poca formulación de preguntas de comprobación en el total de las actividades podría explicarse porque sólo existió una actividad de naturaleza práctica. Con respecto a la actividad contextualizada desde la historia de la ciencia (2), podemos mencionar que hubo una tendencia hacia la formulación de preguntas de explicación causal y preguntas de generalización, esto pues, a pesar de estar desde la historia de la ciencia también se situaba en la comprensión de un fenómeno biológico observable.

Por último, hubo espacios de trabajo metacognitivo con el estudiantado al momento del desarrollo de las actividades, el ¿qué quieres preguntar?, surgió como un continuo tránsito para los estudiantes mientras formulaban sus preguntas, y asimismo, se instalaba un espacio concreto de trabajo en que el estudiantado asumía un rol protagónico en la clase, cuyo trabajo se diseñó para ese objetivo y por tanto, nunca se hizo de manera ingenua. Además, durante la implementación de las actividades no existieron espacios para la enseñanza de la formulación de buenas preguntas y los resultados anteriormente descritos, surgen a partir del constante desarrollo de las actividades durante las sesiones de clase, lo que también indica que proveer de oportunidades y espacios diseñados para que el estudiantado plantee preguntas, mejoraría paulatinamente el nivel de éstas al permitirles pensarlas, practicarlas y motivarse por esto (FURMAN; BARRETO PÉREZ; SANMARTÍ, 2006; SANMARTÍ; MÁRQUEZ; GARCÍA ROVIRA, 2002; ROJAS; JOGLAR, 2017).

\section{Agradecimientos}

Esta investigación se realiza en el marco de la Tesis de Magister de Didáctica de las Ciencias Experimentales de la Pontificia Universidad Católica de Valparaíso, y también fue parte del proyecto de investigación FONDECYT 11150873 de la Universidad de Santiago de Chile.

\section{Referencias}

AGUIAR, O. G.; MORTIMER, E. F.; SCOTT, P. Learning from and responding to students' questions: the authoritative and dialogic tension. Journal of Research in Science Teaching, Hoboken, v. 47, n. 2, p. 74-193, 2010. DOI: http://doi.org/bb8k5g.

AMOS, S. Teachers' questions in the science classroom. In: AMOS, S.; BOOHAM, R. (ed.). Aspects of teaching secondary science: perspectives on practice. London: Routledge, 2002. p. 5-14.

BARELL, J. Aprendizaje basado en problemas: un enfoque investigativo. Buenos Aires: Manantial, 1999. 
BLOSSER, P. How to ask the right questions. Arlington: NSTA Press, 2000.

CAAMAÑO ROS, A. Experiencias, experimentos ilustrativos, ejercicios prácticos e investigaciones: ¿una clasificación útil de los trabajos prácticos? Alambique, Barcelona, n. 39, p. 8-19, 2004.

CAMACHO GONZALEZ, J. P.; QUINTANILLA GATICA, M. Resolución de problemas científicos desde la historia de las ciencia: retos y desafíos para promover competencias cognitivo-lingüísticas en la química escolar. Ciência \& Educação, Bauru, v. 14, n. 2, p. 197-212, 2008. DOI: http://doi.org/cc2vfh.

CAMARGO, A. N. B.; LINDEMEYER, C.; IRBER, C.; RAMOS, M. G. A pergunta na sala de aula: concepções e ações de professores de ciências e matemática. In: ENCONTRO NACIONAL DE PESQUISA EM EDUCAÇÃO EM CIÊNCIAS, 8., 2011, Rio de Janeiro. Atas [...]. Rio de Janeiro: ABRAPEC, 2011. Recuperado el 28 jul. 2020 de: http://tinyurl.com.br/66i.

CHILE. Agencia de Calidad de la Educación. Informe de resultados PISA 2015: competencia científica, lectora y matemática en estudiantes de quince años en Chile. Santiago de Chile: Agencia de Calidad de la Educación, 2016. Recuperado el 28 jul. 2020 de: http://tinyurl.com.br/66j.

CHILE. Ministerio de Educación. Bases curriculares: $7^{\circ}$ basico a $2^{\circ}$ medio: ciencias naturales. Santiago de Chile: MINEDUC, 2013.

CHIN, C. Teacher questioning in science classrooms: approaches that stimulate productive thinking. Journal of Research in Science Teaching, Hoboken, v. 44, n. 6, p. 815-843, 2007. DOI: http://doi.org/ cjq44s.

CHIN, C.; BROWN, D. Student-generated questions: a meaningful aspect of learning in science. International Journal of Science Education, London, v. 24, n. 5, p. 521-549, 2002. DOI: http://doi.org/ fwbjhz.

CHIN, C.; CHIA, L. G. Problem-based learning: using students' questions to drive knowledge construction. Science Education, Hoboken, v. 88, n. 5, p. 707-727, 2004. DOI: http://doi.org/fjxq67.

CHIN, C.; OSBORNE, J. Students' questions: a potential resource for teaching and learning science. Studies in Science Education, Leeds, v. 44, n. 1, p. 1-39, 2008. DOI: http://doi.org/btn5np.

DÍAZ-MORENO, N.; JIMÉNEZ-LISO, M. Las controversias sociocientíficas: temáticas e importancia para la educación científica. Revista Eureka sobre Enseñanza y Divulgación de las Ciencias, Cádiz, v. 9, n. 1, p. 54-70, 2012. Recuperado el 28 jul. 2020 de: http://tinyurl.com.br/66k.

FURMAN, M. G.; BARRETO PÉREZ, M.; SANMARTÍ, N. El procés d'aprendre a plantejar preguntes investigables. Educació Química, Barcelona, v. 14, p. 1-8, 2006. Recuperado el 28 jul. 2020 de: http:// hdl.handle.net/11336/86445.

GRAESSER, A. C.; McMAHEN, C. L.; JOHNSON, B. K. Question asking and answering. In: GERNSBACHER, M. A. (ed.). Handbook of psicolinguistics. New York: Academic, 1994. p. 517-538.

HERNÁNDEZ SAMPIERI, R.; FERNÁNDEZ COLLADO, C.; BAPTISTA LUCIO, P. Metodología de la investigación. México: McGraw Hill, 2010.

IZQUIERDO, M.; ESPINET, M.; GARCÍA, M.P.; PUJOL, R.M.; SANMARTÍ, N. Caracterización y fundamentación de la ciencia escolar. Enseñanza de las Ciencias, Barcelona, p. 79-91, 1999. (Número extra).

JARA CAMPOS, R. Modelos didácticos de profesores de química en formación inicial. Tese (Doctorado en Ciencias de la Educación) - Pontificia Universidad Católica de Chile, Santiago de Chile, 2012. Recuperado el 28 jul. 2020 de: http://tinyurl.com.br/66l. 
JESUS, H. P.; MOREIRA, A. C. The role of students' questions in aligning teaching, learning and assessment: a case study from undergraduate sciences. Assessment \& Evaluation in Higher Education, Bath, v. 34, n. 2, p. 193-208, 2009. DOI: http://doi.org/cjwd3m.

JOGLAR, C. Elaboración de preguntas científicas escolares en clases de biología: aportes a la discusión sobre las competencias de pensamiento científico desde un estudio de caso. Tesis (Doctorado en Ciencias de la Educación) - Pontificia Universidad Católica de Chile, Santiago de Chile, 2014.

JOGLAR, C.; ASTROZA, M.; DE LA FUENTE, R.; CEKALOVIC, P.; ROCCA, A. La membrana plasmática: el desarrollo histórico del modelo y su uso en el aula. In: QUINTANILLA, M.; DAZA, S.; CABRERA, H. Historia y filosofía de la ciencia: aportes para una "nueva aula de ciencias", promotora de ciudadanía y valores. Santiago de Chile: Editorial Bellaterra, 2014. p. 285-304.

LAWSON, A. Science teaching and development of thinking. Belmont: Wadsworth, 2002.

MALVAEZ, O.; JOGLAR, C.; QUINTANILLA, M. Elaboración de preguntas de los estudiantes para promover la metacognición en el aprendizaje activo en ciencias. In: ENCONTRO NACIONAL DE PESQUISA EM EDUCAÇÃO EM CIÊNCIAS, 9., 2013, Águas de Lindóia. Atas [...]. Rio de Janeiro: ABRAPEC, 2013. Recuperado el 28 jul. 2020 de: http://tinyurl.com.br/66m.

MANZI, J.; GONZÁLEZ, R.; SUN, Y. (ed.). La evaluación docente en Chile. Santiago: Pontificia Universidad Católica de Chile, 2011. Recuperado el 28 jul. 2020 de: http://tinyurl.com.br/66n.

MÁRQUEZ BARGALLÓ, C.; ROCA TORT, M. Plantear preguntas: un punto de partida para aprender ciencias. Revista Educación y Pedagogía, Medellín, v. 18, n. 45, p. 61-71, 2006.

MARTÍNEZ GALAZ, C.; GONZÁLEZ WEIL, C. Concepciones del profesorado universitario acerca de la ciencia y su aprendizaje y cómo abordan la promoción de competencias científicas en la formación de futuros profesores de biología. Enseñanza de las Ciencias, Barcelona, v. 32, n. 1, p. 51-81, 2014.

MARTÍNEZ-PÉREZ, L.; PARGA LOZANO, D. La emergencia de las cuestiones sociocientíficas en el enfoque CTSA. Góndola: revista de enseñanza y aprendizaje de las ciencias, Bogotá, v. 8, n. 1, p. 23$35,2013$.

MATTHEWS, M. R. Historia, filosofía, y enseñanza de las ciencias: la aproximación actual. Enseñanza de las Ciencias, Barcelona, v, 12, n. 2, p. 255-277, 1994.

ORGANISATION FOR ECONOMIC CO-OPERATION AND DEVELOPMENT. Assessing scientific, reading and mathematical literacy: a framework for PISA 2006. Paris: OCDE, 2006.

PORTA, L.; SILVA, M. La investigación cualitativa: el análisis de contenido en la investigación educativa. Anuario Digital de Investigación Educativa, Córdoba, n. 14, p. 1-18, 2003. Recuperado el 28 jul. 2020 de: http://tinyurl.com.br/66o.

POZO J. I.; ANGÓN, Y. A solução de problemas como conteúdo procedimental da educação. In: POZO, J. I. A solução de problemas: aprender a resolver, resolver para aprender. Porto Alegre: Artmed, 1998. p. 139-165.

ROCA TORT, M. Las preguntas en el proceso de enseñanza-aprendizaje de las ciencias. Educar, España, v. 33, n. 2, p. 73-80, 2005.

ROCA TORT, M.; MÁRQUEZ BARGALLÓ, C.; SANMARTÍ PUIG, N. Las preguntas de los alumnos: una propuesta de análisis. Ensenanza de las Ciencias, Barcelona, v. 31, n. 1, p. 95-114, 2013. Recuperado el 28 jul. 2020 de: http://tinyurl.com.br/66p.

RODRÍGUEZ, G.; GIL, J.; GARCÍA, E. Metodología de la investigación cualitativa. Málaga: Aljibe, 1999. 
ROJAS, A.; JOGLAR, C. Buenas preguntas del estudiantado en clases de biología, a partir de cuestiones socio-científicas. Enseñanza de las Ciencias, Barcelona, p. 4665-4670, 2017. (Número extra).

SANDÍN, M. Investigación cualitativa en educación. Madrid: Mc Graw Hill, 2003.

SANMARTÍ, N.; MÁRQUEZ, C.; GARCÍA ROVIRA, P. Los trabajos prácticos, punto de partida para aprender ciencias. Aula de Innovación Educativa, Barcelona, v. 113-114, p. 8-13, 2002. Recuperado el 28 jul. 2020 de: https://core.ac.uk/reader/132120670.

SILVESTRI, A. La formulación de preguntas para la comprensión de textos: estudio experimental. Revista Signos, Valparaiso, v. 39, n. 62, p. 493-510, 2006. Recuperado el 28 jul. 2020 de: https:// dialnet.unirioja.es/servlet/articulo?codigo $=2191032$.

SOLBES, J.; TORRES MERCHÁN, N. Y. Análisis de las competencias de pensamiento crítico desde el aborde de las cuestiones sociocientíficas: un estudio en el ámbito universitario. Didáctica de las Ciencias Experimentales y Sociales, Vigo, n. 26, p. 247-269, 2012. Recuperado el 28 jul. 2020 de: http://tinyurl.com.br/66q.

SOLBES, J.; TRAVER, M. Resultados obtenidos introduciendo historia de la ciencia en las clases de física y química: mejora de la imagen de la ciencia y desarrollo de actitudes positivas. Enseñanza de las Ciencias, Barcelona, v. 19, n. 1, p. 151-162, 2001. Recuperado el 28 jul. 2020 de: http://tinyurl. com.br/66r.

TENREIRO-VIEIRA, C.; MARQUES VIEIRA, R. Diseño y validación de actividades de laboratorio para promover el pensamiento crítico de los alumnos. Revista Eureka sobre Enseñanza y Divulgación de las Ciencias, Cádiz, v. 3, n. 3, p. 452-446, 2006. Recuperado el 28 jul. 2020 de: http://tinyurl.com.br/66t.

UNESCO. Aportes para la enseñanza de las ciencias naturales. Santiago: Unesco, 2009. Recuperado el 28 jul. 2020 de: http://tinyurl.com.br/66u.

ZOHAR, A. El pensamiento de orden superior en las clases de ciencias: objetivos, medios y resultados de investigación. Enseñanza de las Ciencias, Barcelona, v. 24, n. 2, p. 157-172, 2008. Recuperado el 28 jul. 2020 de: http://tinyurl.com.br/66v. 